\title{
The Relationship between Public Life Quality and the Voice Handicap Index (VHI) in Adults with Different Hearing Losses
}

\author{
Negin Moradi (PhD) ${ }^{1}$, Parvane Rahimifar (MSc)ㄹ, Samira Aghadoost (PhD) ${ }^{2}$, Majid \\ Soltani (PhD) $)^{1, *}$, Nader Saki (MSc) ${ }^{3}$, Ehsan Naderifar (MSc) ${ }^{1}$ \\ ${ }^{1}$ Musculoskeletal Rehabilitation Research Center,Ahvaz Jundishapur University of Medical Sciences, Ahvaz, Iran \\ ${ }^{2}$ Department of Speech Therapy, School of Rehabilitation, Tehran University of Medical Sciences, Tehran. Iran \\ ${ }^{3}$ Departments of ENT, Imam Hospital, Ahvaz Jondishapour University of Medical Sciences, Ahvaz, Iran Khuzestan Cochlear \\ Implant Center, Ahvaz, Iran
}

* Corresponding Author: Majid Soltani, Musculoskeletal Rehabilitation Research Center,Ahvaz Jundishapur University of Medical Sciences, Ahvaz, Iran. Email: majidsoltanist@gmail.com

\begin{tabular}{|c|c|}
\hline & Abstract \\
\hline $\begin{array}{l}\text { Received: 24/07/2018 } \\
\text { Accepted: 13/02/2019 }\end{array}$ & \multirow{3}{*}{$\begin{array}{l}\text { Background and Objective: In health system using patient-based tools to } \\
\text { measure life quality is a source of making clinical decision. The voice of } \\
\text { individuals with hearing loss faces have some problems because of getting } \\
\text { inappropriate feedback. According to multilateral impacts on life quality, it } \\
\text { is necessary to study the relationship between public life quality (SF } 36 \text { ) } \\
\text { and voice-related life quality (VHI) in adults with different hearing losses. } \\
\text { Materials and Methods: The present study is descriptive-analytical and } \\
\text { samples were } 50 \text { individuals with hearing loss ( } 25 \text { individuals with mild } \\
\text { hearing losses, } 25 \text { individuals with moderate to severe). Basic information } \\
\text { was extracted by interview and then the subjects completed the SF36 and } \\
\text { VHI questionnaires. The mean score of each questionnaire was calculated, } \\
\text { and the obtained data were analyzed by spearman statistical test in SPSS } 22 \\
\text { software. } \\
\text { Results: There isn't any significant correlation between the total score and } \\
\text { three subtests of VHI (emotional, physical, and functional) and SF36 in } \\
\text { individuals with mild hearing loss. Also the results showed that there is } \\
\text { significant correlation between the total score and two subtests of VHI } \\
\text { (physical and functional) except emotional tests and SF36 in individuals } \\
\text { with moderate to severe hearing loss. } \\
\text { Conclusion: This study emphasizes the need for psychosocial rehabilitation } \\
\text { along with sound technique. So it is necessary that speech and language } \\
\text { pathologists try to improve cognitive-psychosocial factors along with voice } \\
\text { treatments to increase voice-related quality of life in these patients. } \\
\text { Keywords: Hearing Loss; Quality of Life; Voice }\end{array}$} \\
\hline $\begin{array}{l}\text { How to Cite this Article: } \\
\text { Moradi N, Rahimifar P, } \\
\text { Aghadoost S, Soltani M, Saki N, } \\
\text { Naderifar E. The Relationship } \\
\text { between Public Life Quality } \\
\text { and the Voice Handicap Index } \\
\text { (VHI) in Adults with Different } \\
\text { Hearing Losses. Pajouhan } \\
\text { Scientific Journal. 2019; 17(2): } \\
\text { 1-6. DOI: } 10.29252 / \text { psj.17.2.1 }\end{array}$ & \\
\hline & \\
\hline
\end{tabular}

Copyright (C) 2019 Pajouhan Scientific Journal. This is an open-access article distributed under the terms of the Creative Commons Attribution-NonCommercial 4.0 International License (http://creativecommons.org/licenses/by-nc/4.0/) which permits copy and redistribute the material just in noncommercial usages, provided the original work is properly cite. 


\section{رابطه كيفيت زندكى با شاخص معلوليت صوتى در افراد بزرتسال با افت هاى مختلف}

\section{نغَين مرادى'، يروانه رحيمى فر '، سميرا آقادوست'، مجيد سلطانى ا."، نادر صاكى'، احسان نادرى فر'}

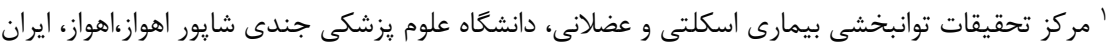

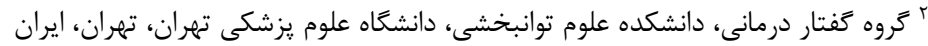

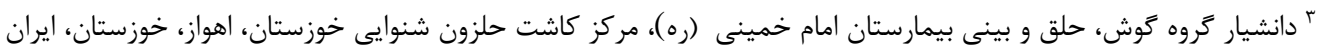
* نويسنده مسئول: مجيد سلطانى، مركز تحقيقات توانبخشى بيمارى اسكلتى و عضلانى، دانشكاه علوم يزشكى جندى شايور اهواز،اهواز، ايران. ايميل: majidsoltanist@gmail.com

\begin{tabular}{|c|c|}
\hline 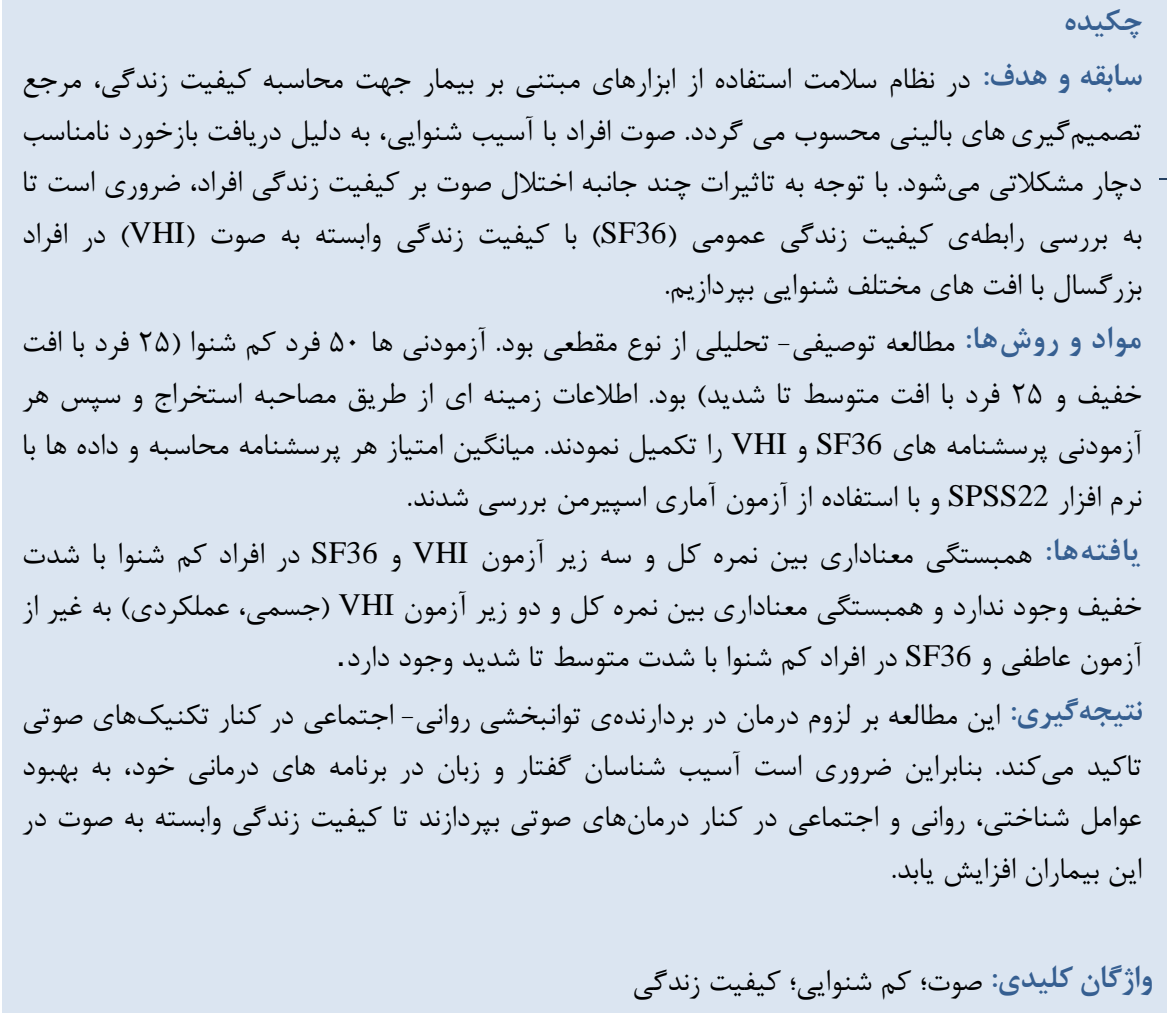 & 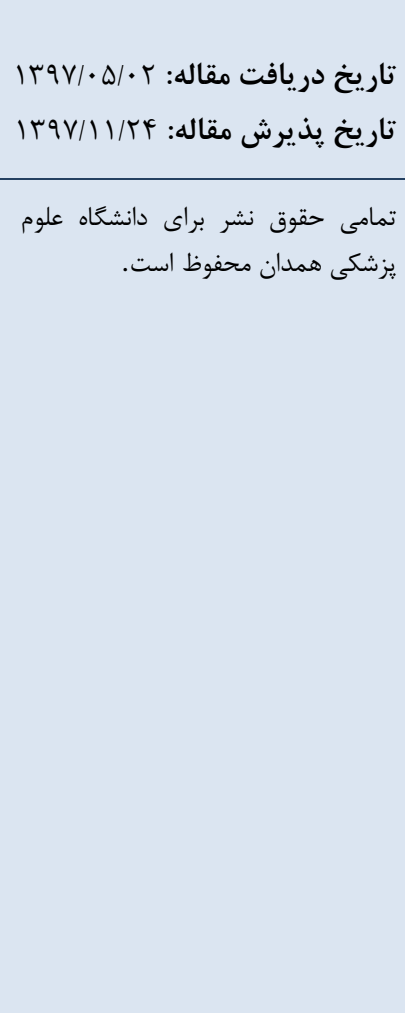 \\
\hline
\end{tabular}

واكهها و همخوانها در اين افراد مشاهده مىشود، كه بر وضـوح

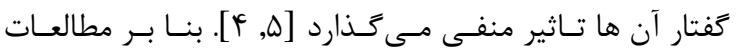

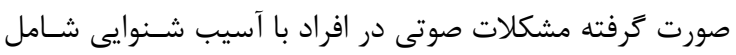

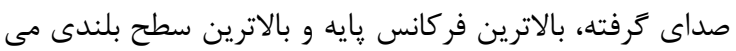

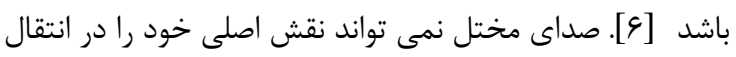

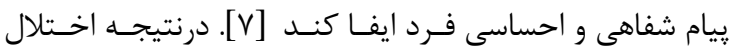

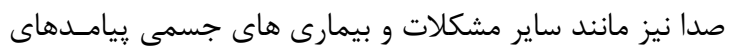

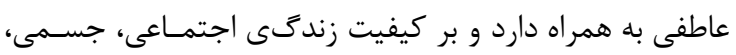

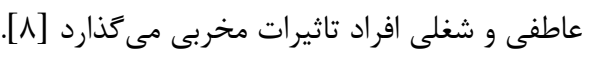

انسان موجودى اجتماعى است و برقرارى ارتباط با ديخـران

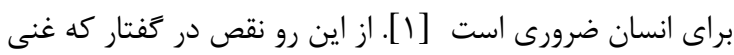

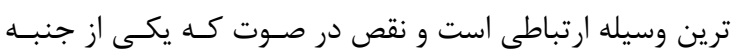

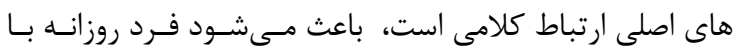

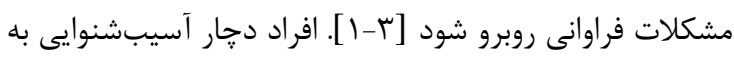

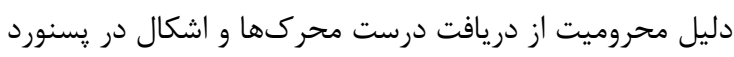

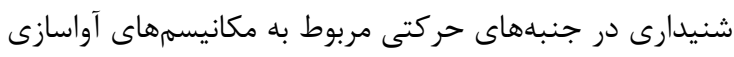

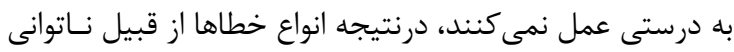

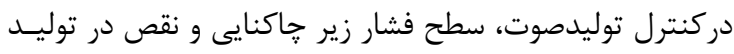


كَفتاردرمانى با تاكيد بر اهميت تاثير اخــتلال صـدا بــر كيفيـت

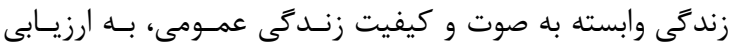

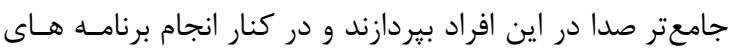
درمانى صوت به برنامه هايى جهت بهبود كيفيـت زنـدكى ايـن

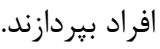

\section{مواد و روشها}

روش انجام اين يزوهش توصيفى - تحليلى، از نــوع مقطعى إنى

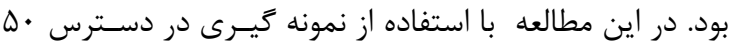

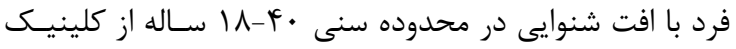

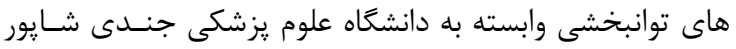

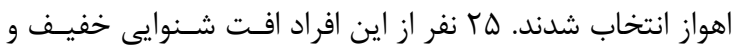

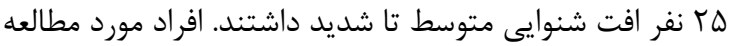

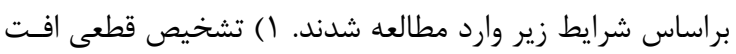

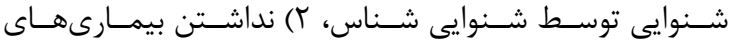

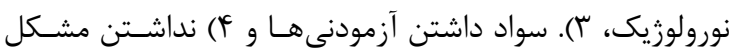
بينايى اصلاح نشده.

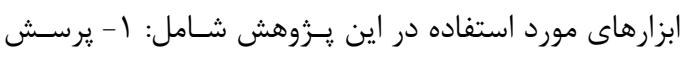

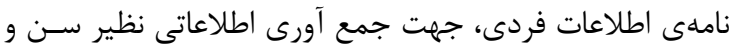

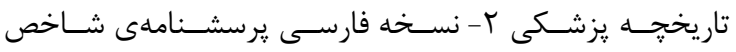

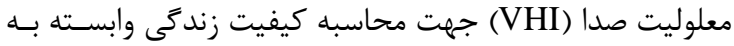

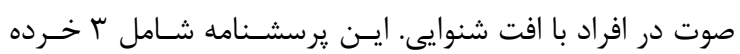

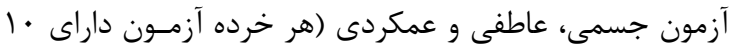

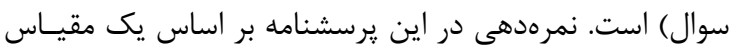

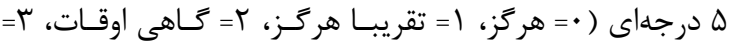

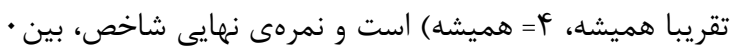

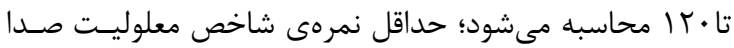

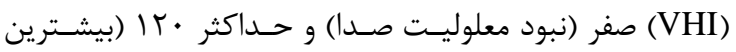

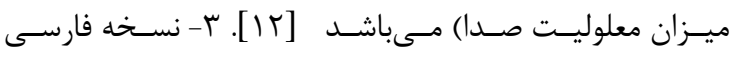

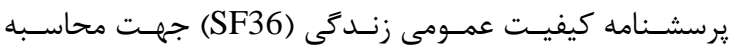

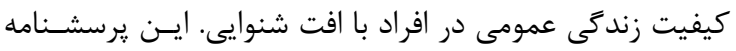

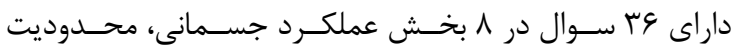

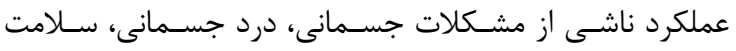

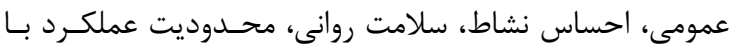

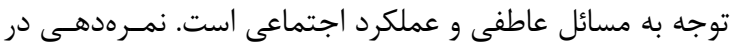

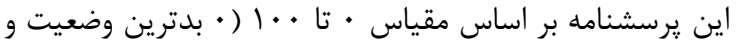

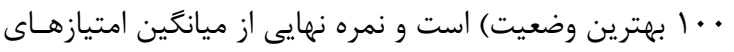

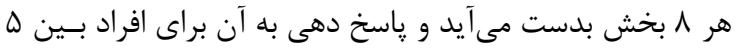

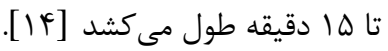

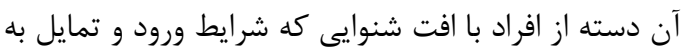

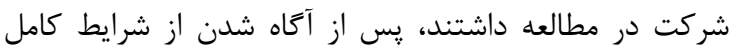

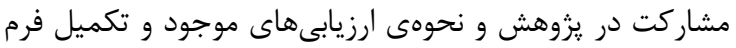

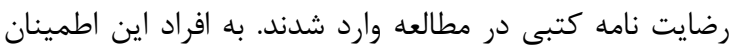
داده شد كه اطلاعات آن ها كاملا محرمانه باقى خواهد ماند ماند و سورد
دو ديدَاه براى ارزيابى كيفيت زندگى وابسته بـهـ سـلامتى

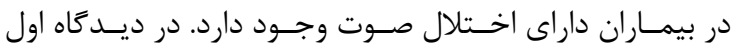

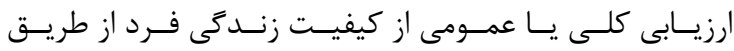

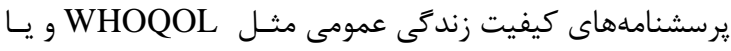

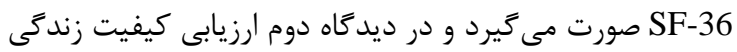

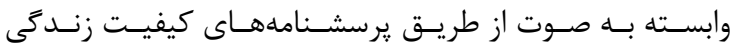

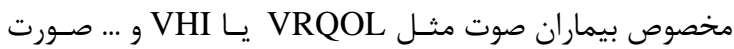

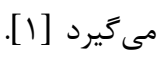
(VHI) Voice Handicap Index

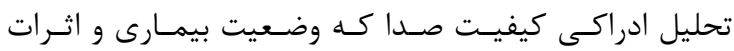

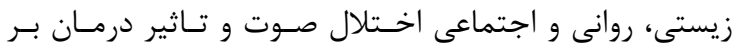

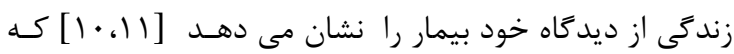

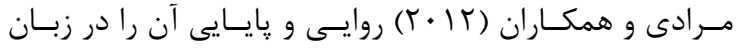

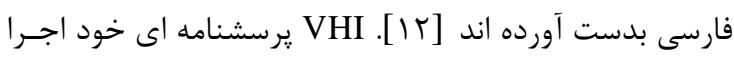

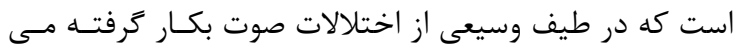

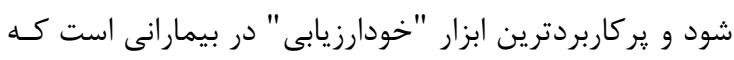

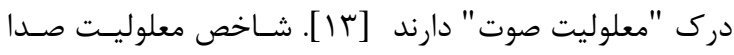

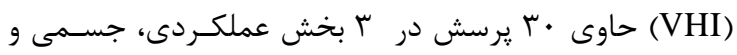

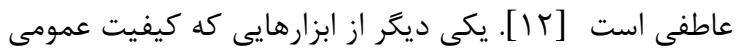

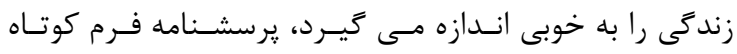

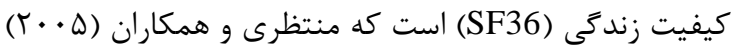

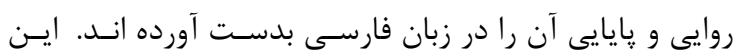

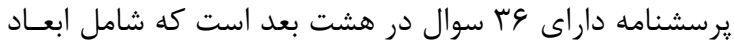

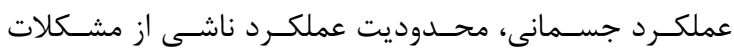

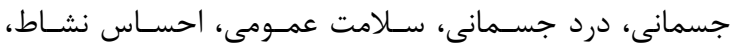

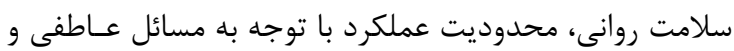

عملكرد اجتماعى مىباشد [IY]

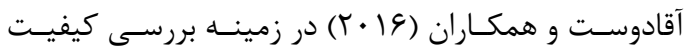

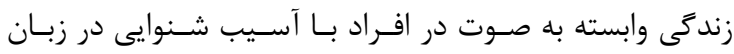

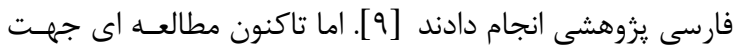

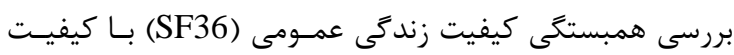

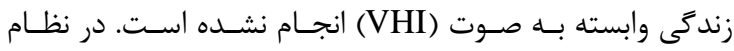

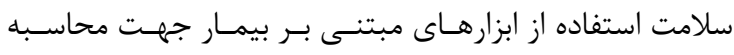

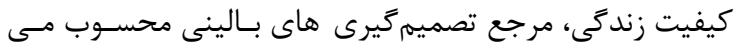

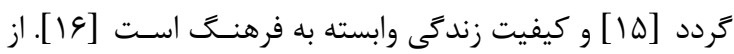

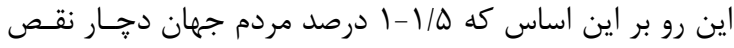

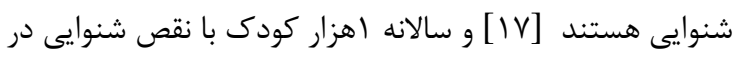

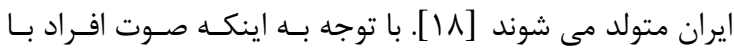

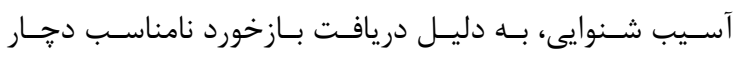

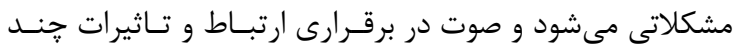

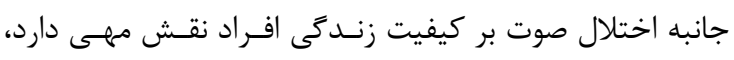

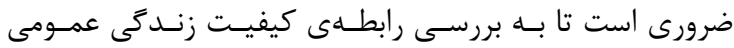

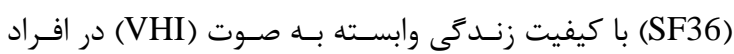
بزركسال با افت هاى مختلف شــوايى بيــردازيمم تـا متخصصـان 
شنوايى خفيف، له نفــر افـت شـنوايى متوسـط تـا شـديد) در

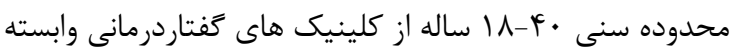

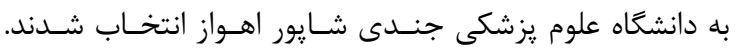

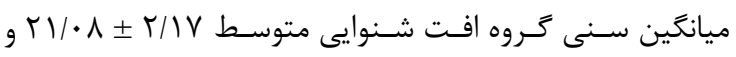

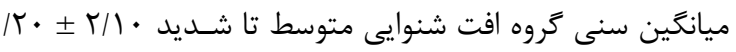

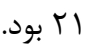
بررسى توزيع داده ها با آزمون كولموكروف-اسييرنوف نشان

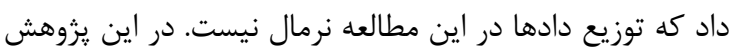

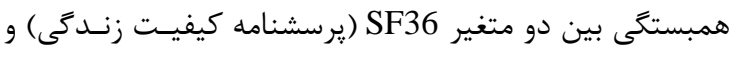
خفر VHI خفيف و افراد با افت شنوايى متوسط تا شديد مورد بررسى قرار

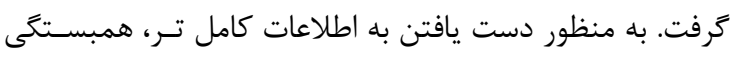

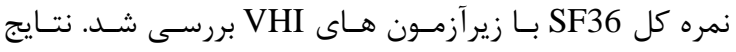

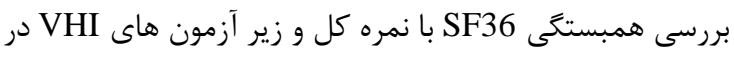
كروه افت شنوايى خفيف و كروه افت شنوايى متوسط تا شـديد در جدول ا و جدول r آمده است.
هر زمان خواستند، مى توانند از مطالعه خارج شوند و هيج

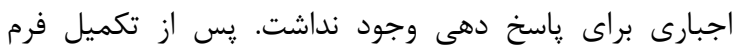

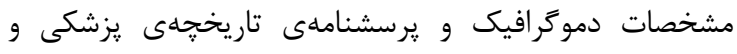

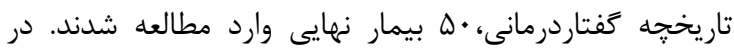

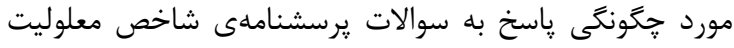

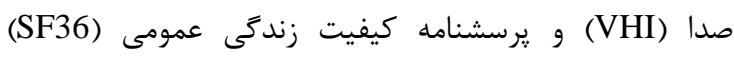

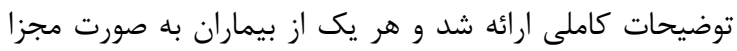

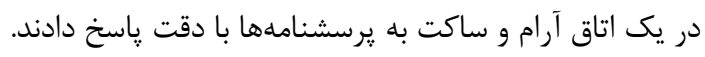

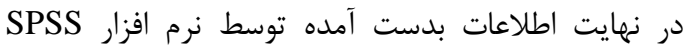

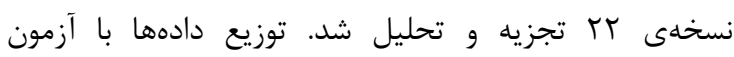

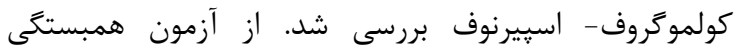

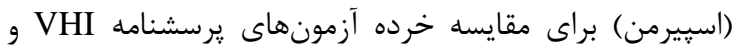

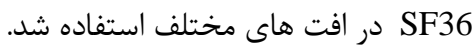

بافتهها در اين مطالعه • له فرد با افت شنوايى (لا نفر از افراد افـت

جدول ا: همبستكى نمره كيفيت زندكى با نمره كل و زير آزمون هاى معلوليت صوتى در افراد كم شنوا با شدت خفيف

\begin{tabular}{|c|c|c|}
\hline مقدار احتمال(P) & ضر يب همبستگى (r) & \\
\hline$\cdot / \pi \Delta \cdot$ & .119. & شاخص معلوليت صوتى \\
\hline$\cdot / 1 \mathrm{~V}$ & $\cdot / r \Lambda$. & عاطفى \\
\hline .119 & $-\cdot \pi \varphi$. & جسمى \\
\hline$\cdot / 1 \Delta$ & $\cdot / r q$. & عملكردى \\
\hline
\end{tabular}

جدول r: همبستكى نمره كيفيت زندكى با نمره كل و زير آزمون هاى معلوليت صوتى در افراد كم شنوا با شدت متوسط تا شديد

\begin{tabular}{|c|c|c|}
\hline مقدار احتمال(P) & ضريب همبستغى (r) & \\
\hline$\cdot / \cdot 4$. & $-\cdot / 4 \cdot$. & شاخص معلوليت صوتى \\
\hline$\cdot / 1 \Delta \cdot$ & $-\cdot / r q$. & عاطفى \\
\hline$\cdot 1 \cdot \Delta$ &.$- / F T$. & جسمى \\
\hline $.1 \cdot 1$. & $-\cdot / \& V$. & عملكردى \\
\hline
\end{tabular}

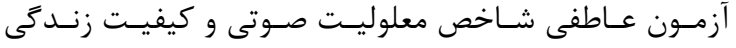

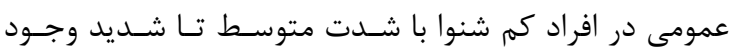
ندارد.

از آنجايى كه صدا داراى ابعاد مختلفى از قبيل بعد عاطفى،

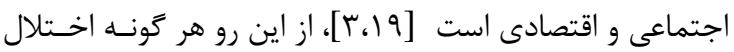

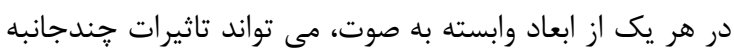

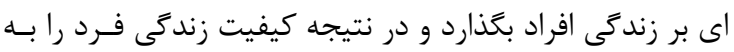

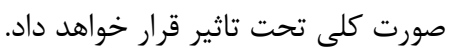

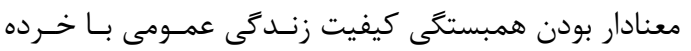

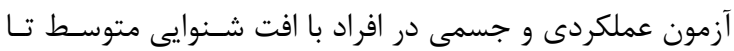

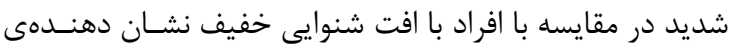

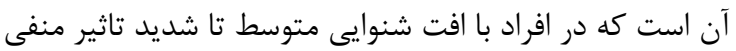

نتايج اين مطالعه نشان داد كه همبستكى معنــادارى بـين

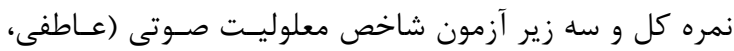

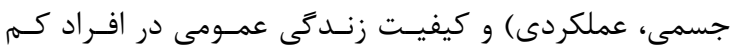

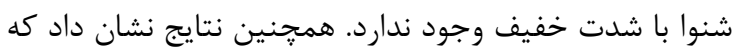

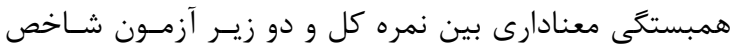

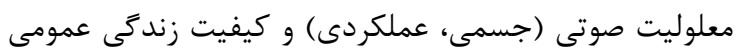

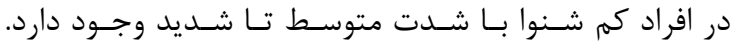

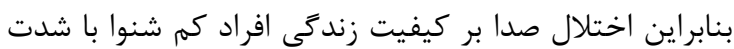

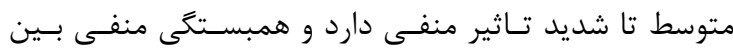

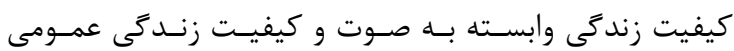

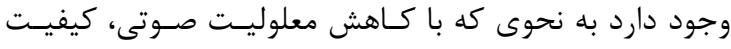

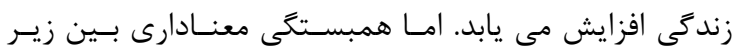


يابد [rr]

$$
\begin{aligned}
& \text { نتيجه كيرى }
\end{aligned}
$$

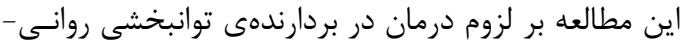

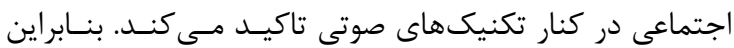

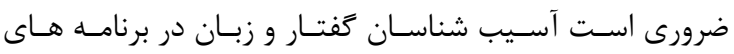

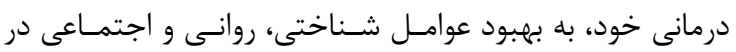

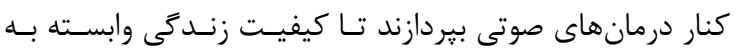

$$
\begin{aligned}
& \text { صوت در اين بيماران افزايش يابد. }
\end{aligned}
$$

$$
\text { تشكر و قدر دانى إن مقاله }
$$

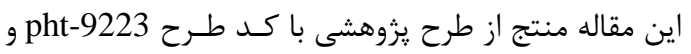

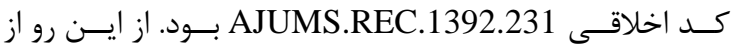

حمايت مركز تحقيقات توانبخشى عضـانلى -اسـكلتى، دانشــاه

$$
\text { علوم يزشكى جندى شايور اهواز قدردانى مى شود. }
$$

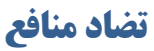

اين مطالعه براى نويسندكان هيجَّونه تضاد منافعى نداشته

\section{REFERENCES}

1. Crannell K. Voice and articulation: Nelson Education; 2011.

2. Nicolosi L, Harryman E, Kresheck J. Terminology of communication disorders: Speech-language-hearing: Lippincott Williams \& Wilkins; 2004.

3. Kent RD. The uniqueness of speech among motor systems. Clinical linguistics \& phonetics. 2004;18(6-8):495-505. DOI: $10.1080 / 02699200410001703600$

4. Angelocci AA, Kopp GA, Holbrook A. The vowel formants of deaf and normal-hearing eleven-to fourteen-year-old boys. Journal of Speech and Hearing Disorders. 1964; 29(2):156-70. DOI.10.1044/jshd.2902.156

5. Hudgins CV, Numbers FC. An investigation of the intelligibility of the speech of the deaf. Genetic psychology monographs. 1942;25:289-392.

6. Aghadoost O, Moradi N, Dabirmoghaddam P, Aghadoost A, Naderifar E, Dehbokri SM. Voice Handicap Index in Persian Speakers with Various Severities of Hearing Loss. Folia Phoniatrica et Logopaedica. 2016;68(5):211-5. DOI: 10.1159/000455230.

7. Malki KH, Mesallam TA, Farahat M, Bukhari M, Murry T. Validation and cultural modification of Arabic voice handicap index. European Archives of Oto-RhinoLaryngology. 2010;267(11):1743-1751

8. Woisard V, Bodin S, Yardeni E, Puech M. The voice handicap index: correlation between subjective patient response and quantitative assessment of voice. Journal of Voice. 2007;21(5):623-31.

9. Hummel C, Scharf M, Schuetzenberger A, Graessel E, Rosanowski F. Objective voice parameters and selfperceived handicap in dysphonia. Folia Phoniatrica et Logopaedica. 2010;62(6):303-7. DOI: 10.1159/000287715

10. Maertens K, de Jong F. The voice handicap index as a tool for assessment of the biopsychosocial impact of voice problems. B-ent. 2006;3(2):61-6.

11. Li H, Xu W, Han D, Hu R, Hu H, Hou L. Self-assessment characteristics of voice handicap index for voice disorders and its influencing factors. Chinese journal of otorhinolaryngology head and neck surgery. 2009;44(2): 109-13.

12. Moradi N, Pourshahbaz A, Soltani M, Javadipour S,

$$
\begin{aligned}
& \text { صوت در عملكرد روزانه بيشتر احساس مى شود كـه منجــر بــهـ } \\
& \text { كاهش كيفيت زندگى عمومى شان شده است. }
\end{aligned}
$$

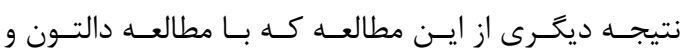

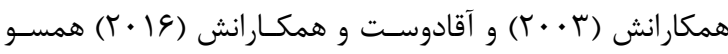

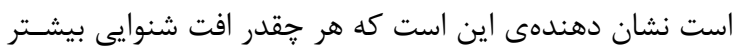

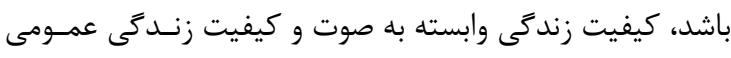

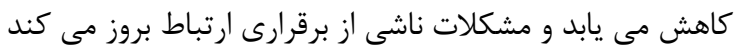

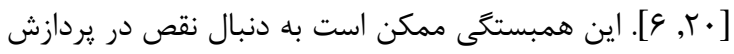

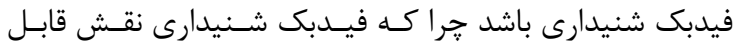

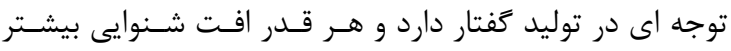

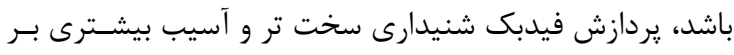

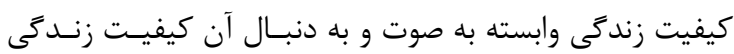

$$
\begin{aligned}
& \text { عمومى دارد [ع] كيفيت زندكي } \\
& \text { از آنجا كه معلوليت به معناى بروز الحمد محدوديت در انجام فعاليت }
\end{aligned}
$$

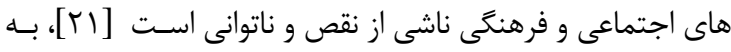

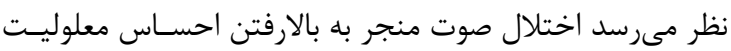

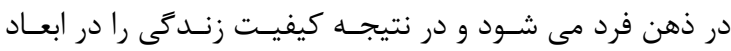

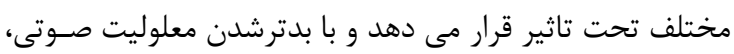

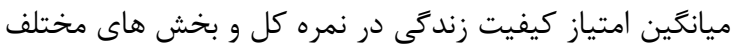

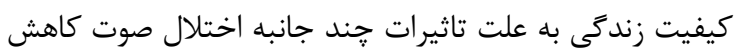

Hashemi H, Soltaninejad N. Cross-cultural equivalence and evaluation of psychometric properties of voice handicap index into Persian. Journal of Voice. 2013;27(2):258. e15e22. DOI: 10.1016/j.jvoice.2012.09.006

13. Behlau M, Alves dos Santos LdM, Oliveira G. Crosscultural adaptation and validation of the voice handicap index into Brazilian Portuguese. Journal of Voice. 2011;25(3):354-359. DOI: 10.1016/j.jvoice.2009.09.007.

14. Montazeri A, Goshtasebi A, Vahdaninia M, Gandek B. The Short Form Health Survey (SF-36): translation and validation study of the Iranian version. Quality of life research. 2005;14(3):875-82.

15. Dabirmoghaddam P, Aghadoost O, Baziar M, Aghadoost A. Comparison voice handicap index (VHI) in patients with vocal mass lesions in larynx and healthy people. Journal of Modern Rehabilitation. 2016;9(7):20-7.

16. Moradi N, Pourshahbaz A, Soltani M, Javadipour S. Cutoff point at voice handicap index used to screen voice disorders among persian speakers. Journal of Voice. 2013;27(1):130. e1-. e5. DOI: 10.1016/j.jvoice.2012.08.007.

17. Mathers C, Smith A, Concha M. Global burden of hearing loss in the year 2000. Global burden of Disease. 2000;18(4):1-30.

18. Sa'adati-Boroujeni S, Hatami-Zadeh N, Vameghi R, Kraskian A. Developing and Validating a Hearing-Loss Related Quality of Life Questionnaire for Adolescents. Archives of Rehabilitation. 2013;13(0):84-93.

19. Kahane JC. Growth of the human prepubertal and pubertal larynx. Journal of Speech, Language, and Hearing Research. 1982;25(3):446-455. DOI.org/10.1044/jshr. 2503.446

20. Dalton DS, Cruickshanks KJ, Klein BE, Klein R, Wiley TL, Nondahl DM. The impact of hearing loss on quality of life in older adults. The gerontologist. 2003;43(5): 661-668.

21. Rosenbaum P, Stewart D, editors. The World Health Organization International Classification of Functioning, Disability, and Health: a model to guide clinical thinking, practice and research in the field of cerebral palsy. Seminars in pediatric neurology.2004;11(1):5-10. 
22. Aghadoost O, Amiri Shavaki Y, Moradi N, Jalayi S, Soltani M, Naderifar E, et al. Correlation between general quality of life with voice related quality of life in female teachers with voice complaint in elementary schools of Tehran. Speech and Language Pathology. 2013; 1(1):44-51. 